\title{
Response to hepatitis B vaccination in patients with inflammatory bowel disease: a prospective observational study in Korea
}

\author{
Ji Young Chang, Sung-Ae Jung, Chang Mo Moon, Seong-Eun Kim, Hye-Kyung Jung, Ki-Nam Shim \\ Department of Internal Medicine, Ewha Womans University School of Medicine, Seoul, Korea
}

Background/Aims: Testing for hepatitis B virus (HBV) serologic markers and appropriate vaccination are required in the management of inflammatory bowel disease (IBD) patients. We evaluated immunogenicity for HBV in IBD patients and the response to the HBV vaccination. Methods: From May 2014 to August 2016, patients diagnosed with IBD were prospectively included and evaluated for antibody to hepatitis B surface antigen, antibody to hepatitis B core antigen, and antibody to hepatitis B surface antigen. Among the 73 patients who were confirmed with nonimmunity to HBV, 44 patients who had completed the 3-dose $\mathrm{HBV}$ vaccination series received a single booster vaccination, while 29 patients who had not completed the vaccinations series or were unsure of receiving the vaccination received a full vaccination series. Results: An optimal response was obtained in $70.5 \%$ of the patients in the booster group, and $89.7 \%$ of the patients in the full vaccination group. Age younger than 26 years (odds ratio [OR], 6.01; 95\% confidence interval [CI], 1.15-31.32; $P=0.033$ ) and a complete previous vaccination series (OR, $0.15 ; 95 \%$ CI, $0.03-0.80 ; P=0.026)$ were associated with optimal vaccine response. Previous complete vaccination series (OR, $0.11 ; 95 \%$ CI, $0.02-0.73 ; P=0.022$ ) was the only predictive factor for lower compliance. Conclusions: The response to the HBV vaccination was lower in patients older than 26 years and for those patients with a complete vaccination history. Since patients with a complete vaccination history also had poor compliance, serum HBV-titers should be checked more thoroughly, and a full vaccination series should be administered in cases when there is a negative response to the booster vaccination. (Intest Res 2018;16:599-608)

Key Words: Hepatitis B virus; Vaccination; Inflammatory bowel diseases

\section{INTRODUCTION}

Patients with IBD are at increased risk of infectious disease raised from the nature of disease, immunosuppressive agents such as biologics and immunomodulators, and procedures including endoscopy, transfusion, and surgery, which are frequently required during treatment., ${ }^{1,2}$ Infectious diseases often lead to higher morbidities and mortalities in

Received January 29, 2018. Revised April 27, 2018.

Accepted May 25, 2018

Correspondence to Sung-Ae Jung, Department of Internal Medicine, Ewha

Womans University School of Medicine, 1071 Anyangcheon-ro, Yangcheon-

gu, Seoul 07985, Korea. Tel: +82-2-2650-2945, Fax: +82-2-2655-2076,

E-mail: jassa@ewha.ac.kr
IBD patients who are generally in an immune-suppressed status. Therefore, effective vaccinations against these diseases should be administered.

HBV infection has been reported to cause fulminant or fatal hepatitis by opportunistic infection or reactivation of $\mathrm{HBV}$ in IBD patients, but is preventable with a vaccine. ${ }^{3-6}$ The American College of Gastroenterology (ACG) clinical guidelines recommend HBV vaccination for every IBD patient lacking HBV immunity with non-immunization regardless of immunosuppression status, especially before the start of anti-tumour necrosis factor (anti-TNF) therapy. ${ }^{7}$ Since antiHBs levels decline over time, ${ }^{8}$ ACG guidelines also recommend a regular check of anti-HBs titer to confirm immunity. ${ }^{7}$

๑ Copyright 2018. Korean Association for the Study of Intestinal Diseases. All rights reserved.

This is an Open Access article distributed under the terms of the Creative Commons Attribution Non-Commercial License (http://creativecommons.org/licenses/by-nc/4.0)

which permits unrestricted non-commercial use, distribution, and reproduction in any medium, provided the original work is properly cited. 
A booster vaccination or a new vaccination series is recommended for patients with insufficient protective antibody concentrations. $^{7}$

As a part of the National Immunization Program, all neonates have received a universal HBV vaccine since 1995 in Korea, regardless of maternal HBsAg status. ${ }^{9}$ With the implementation of the national vaccination program, $\mathrm{HBsAg}$ prevalence has decreased from $10 \%$ in the $1980 \mathrm{~s}^{10}$ to $2.9 \%$ in $2013 .{ }^{11}$ However, it has been reported that the decline of $\mathrm{HBV}$ infection is limited to the younger population whereas the prevalence of $\mathrm{HBV}$ infection in the middle-aged and elderly populations has remained unchanged. ${ }^{9} \mathrm{HBV}$ infection is still the leading cause of chronic liver disease, including chronic hepatitis, cirrhosis, and hepatocellular carcinoma. ${ }^{12}$ Therefore, it is important to monitor the status of HBV immunity and vaccinate IBD patients who lack HBV immunity.

In this study, we aimed to assess the following: (1) the HBV immune status in patients with IBD; (2) the response to a booster $\mathrm{HBV}$ vaccination in patients who had previous completed a 3-dose vaccination series or the response to a 3-dose HBV vaccination series in patients with incomplete or an unknown vaccination history; (3) the predictive factors for an effective HBV vaccine response; (4) the response to a new complete vaccination series (revaccination) in patients who had shown no response to a booster vaccination; and (5) the factors associated with compliance to the recommended vaccination schedule.

\section{METHODS}

\section{Study Subjects and Enrollment}

Patients from the IBD clinic in Ewha Womans University Mokdong Hospital who provided informed consent were enrolled prospectively from May 2014 to August 2016. After informed consent was obtained, peripheral blood samples were collected to evaluate HBV serologic markers (anti-HBs, HBsAg, and anti-HBc).

Patients who were diagnosed with IBD by clinical, endoscopic, radiographic, and histological assessment and older than 15 years at the time of screening were included. Subjects with severe comorbidities including liver cirrhosis, chronic renal failure, and malignant disease were excluded. For the analysis of vaccine response and response associated factors, only the patients who were confirmed with negative serologic results for anti-HBc, and anti-HBs, and negative serologic result for HBsAg were included.

Prior HBV infection included resolved HBV infection or remote infection, and each category was defined by the pres- ence of anti-HBc and anti-HBs or the presence of anti-HBc without anti-HBs, respectively. Present HBV infection, which included carrying an inactive HBsAg, and chronic hepatitis B were defined as the presence of HBsAg. Immunity after prior vaccination was defined as the presence of anti-HBs without anti-HBc. ${ }^{2,13,14}$ The Institutional Review Board of Ewha Womans University Hospital approved this study (IRB No. 201406-026-008).

\section{Study Design}

The vaccine used in this study was the Hepavax-Gene $\mathrm{TF}^{\circledR}$ (purified hepatitis B type surface antigen, $1.0 \mathrm{~mL}, 20 \mu \mathrm{g}$ ) manufactured by Green Cross Corp (Yongin, Korea). The vaccine was injected into the deltoid muscle.

Among the patients who were confirmed with nonimmunity to HBV, patients who had been previously vaccinated with a 3-dose HBV vaccination series received a booster vaccination (booster group), and the patients who had not completed the hepatitis B vaccination series or were unsure of their vaccination history were administered a 3-dose HBV vaccination in months 0,1 , and 6 (full series vaccination group). A new complete 3-dose vaccination was administered to patients who did not acquire adequate immune response with a booster vaccination. All patients were instructed on possible adverse events related to the HBV vaccination and to report their symptoms to the study staff as soon as possible.

\section{Study Outcomes}

\section{1) Vaccination Compliance}

Good compliance was defined as patients who had received every recommended vaccination. Only those patients who had shown good compliance were included in the evaluation of vaccine response.

\section{2) Assessment of Vaccine Response}

The serum anti-HBs level was measured at least 1 month after either the booster vaccination or the last of the 3-dose vaccination series. The response was considered as optimal if the anti-Hbs titer was equal to or higher than $10 \mathrm{IU} / \mathrm{L}$. These responses were sub-categorized using different antiHBs cutoff points: effective immune response (EIR, anti-Hbs $\geq 100 \mathrm{IU} / \mathrm{L}$ ) and adequate immune response (AIR, anti-Hbs $\geq$ $10 \mathrm{IU} / \mathrm{L}$ ). A serum anti-HBs level below $10 \mathrm{IU} / \mathrm{L}$ was considered as non-responsive. ${ }^{1}$

\section{Demographic and Clinical Data Collection}

The following demographic and clinical data were collected 
to analyze predictive factors associated with vaccine response and compliance: demographic information (age at the first dose vaccination in this study and sex), comorbidities, IBD subtype, disease duration (interval between IBD diagnosis and vaccination), disease severity, treatment at the time of first dose vaccination in this study, and the indication for vaccination. Disease severity was assessed using a partial Mayo score (if $<5$, mild; if $5-7$, moderate; if $>7$, severe) for $\mathrm{UC}^{15}$ and a CDAI (if 150-219, mild; if 220-450, moderate; if $>450$, severe $)^{16}$ for CD. The types of treatment were categorized into 3 group: (1) biologics group, which includes infliximab, adalimumab, golimumab, or any combination with biologics; (2) an immunosuppressive group, which includes corticosteroids, azathioprine, methotrexate, cyclosporine A, or any combination of these drugs with aminosalicylates; and (3) a non-immunosuppressive group (only aminosalicylate).

\section{Statistical Analysis}

All statistical analyses were carried out using the SPSS program, version 22.0 (IBM Corp., Armonk, NY, USA). Continuous variables are presented as the mean with standard deviation, and categorical variables are presented as number with percentage. In univariate analyses, Student $t$-test was used for continuous variables, and the chi-square test, Fisher exact test, or linear-by-linear association was used for categorical variables. Multivariate logistic regression analysis was used to identify independent predictive factors for optimal vaccine response and compliance. $P$-values $<0.05$ were considered statistically significant.
Table 1. Baseline Characteristics of the Vaccinated Patients ( $n=73$ )

\begin{tabular}{|c|c|}
\hline Characteristics & Value \\
\hline Age at the time of HBV vaccination (yr) & $29.9 \pm 12.3$ \\
\hline Disease duration (mo) & $56.8 \pm 61.6$ \\
\hline Male sex & $49(67.1)$ \\
\hline \multicolumn{2}{|l|}{ IBD subtype } \\
\hline UC & $28(38.4)$ \\
\hline$C D$ & $45(61.6)$ \\
\hline \multicolumn{2}{|l|}{ Disease severity } \\
\hline Remission to mild & $60(82.2)$ \\
\hline Moderate to severe & $13(17.8)$ \\
\hline \multicolumn{2}{|l|}{ Type of treatment } \\
\hline Biologics + immunomodulator & $10(13.7)$ \\
\hline Biologics $^{a}$ & $13(17.8)$ \\
\hline Immunomodulator $^{\mathrm{b}}$ & $28(38.4)$ \\
\hline Non-immunomodator ${ }^{c}$ & $22(30.1)$ \\
\hline \multicolumn{2}{|l|}{ Comorbidity } \\
\hline None & $67(91.8)$ \\
\hline Diabetes mellitus & $2(2.7)$ \\
\hline Hypertension & $3(4.1)$ \\
\hline Pancreatitis & $1(1.4)$ \\
\hline \multicolumn{2}{|l|}{ Indication for HBV vaccination } \\
\hline Booster after complete HBV vaccination & $44(60.3)$ \\
\hline Incomplete vaccination or unknown history & $29(39.7)$ \\
\hline
\end{tabular}

Values are presented as mean \pm SD or number (\%).

aiologics included infliximab, adalimumab, golimumab, or any combination of these drugs with aminosalicylates.

${ }^{b}$ Immunomodulator included corticosteroids, thiopurine, or any combination of these drugs with aminosalicylates.

cNon-immunomodulator included aminosalicylate.

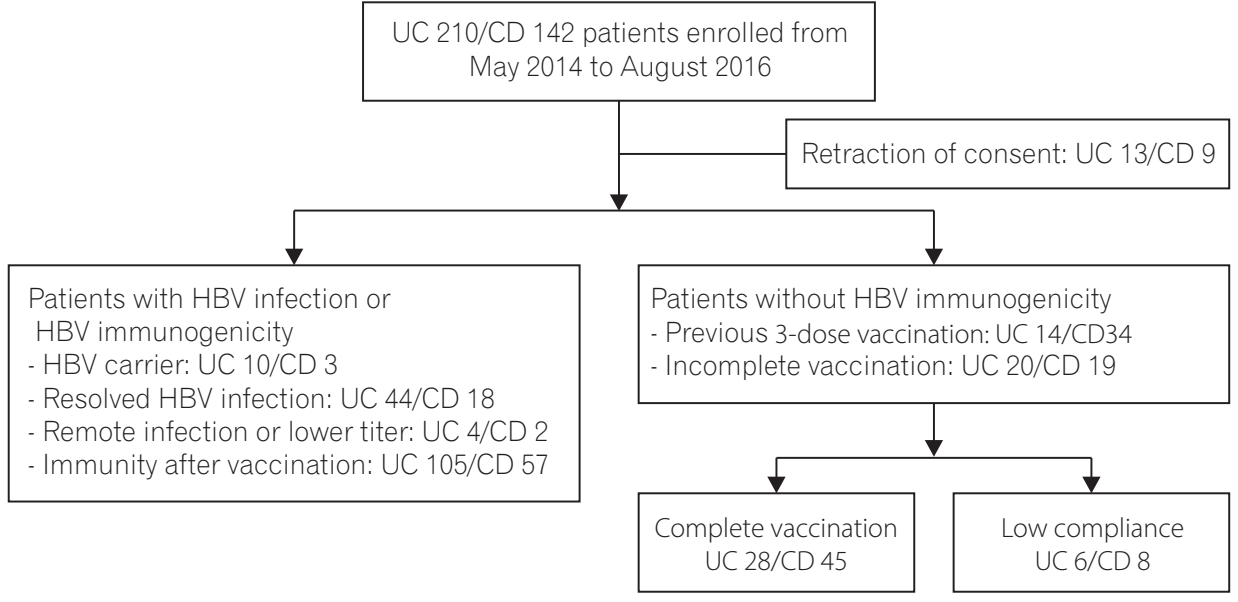

Fig. 1. Flowchart of enrolled patients. 


\section{RESULTS}

\section{Baseline Characteristics}

A total of 352 patients with IBD (210 UC, $142 \mathrm{CD}$ ) were enrolled in this study. After excluding 22 patients who retracted the consent, (UC 13, CD 9), a total of 330 patients were included in this study (Fig. 1). The mean age of the patients was 38.5 years, and $62.7 \%$ of patients were male. One hundred and ninety-seven patients $(59.7 \%)$ were diagnosed with UC, and 133 (40.3\%) were diagnosed with CD. Regarding the HBV immune status, 81 patients (24.5\%) had prior or present HBV infection (13 HBV carrier [UC 10, CD 3], 62 resolved HBV infection [UC 44, CD 18], 6 remote HBV infection [UC 4, CD 2]), 162 patients (49.1\%) had immunity after prior vaccination (UC 105, CD 57), and 87 patients (26.4\%) did not have immunity to HBV (UC 34, CD 53).

Among 87 patients who had nonimmunity to HBV and were decided to receive $\mathrm{HBV}$ vaccination, 14 patients who showed low compliance to vaccination were excluded for further analysis. Baseline characteristics of vaccinated patients are shown in Table 1. The mean age at the time of initial vaccination in this study was 29.9 years, and $67.1 \%$ of patients were male. Sixty patients $(82.2 \%)$ had maintained disease activity from remission to mild status under the prescribed medication. Regarding the indication for HBV vaccination, 44 patients with a previous complete HBV vaccination (60.3\%; UC 13, CD 31) received a booster vaccination, while 29 patients without prior vaccination or an unknown history of vaccination (39.7\%; UC 15, CD 14) received a full 3 -dose vaccination. There were no severe adverse events related to vaccination during the study.

\section{Response to Vaccination}

Among the 44 patients who received a booster vaccination, 31 (70.5\%) obtained optimal responses. There were no significant differences between optimal vaccine response and disease type (53.8\% UC and $77.4 \%$ CD, respectively, $P=0.155)$. Among the 29 patients who received the full HBV vaccination series, 26 (89.7\%) showed optimal responses. There was also no significant difference between optimal vaccine response and disease type (93.3\% UC and $85.7 \% \mathrm{CD}$, respectively, $P=0.598$ ) (Fig. 2).

When vaccine responses were analysed, the EIR and AIR of UC patients were slightly lower than in CD patients in the booster vaccination group, whereas the EIR and AIR of UC patients were higher than in CD patients in the full vaccination group. However, these differences were not statistically

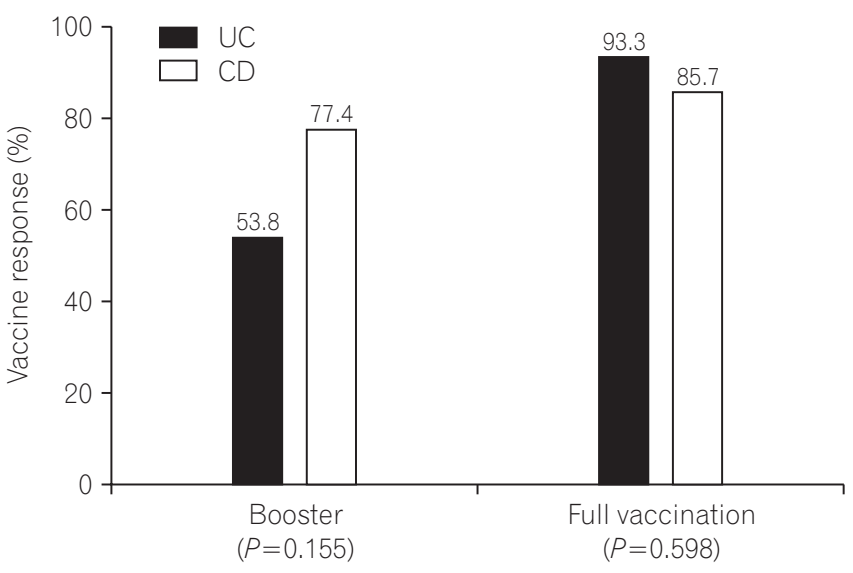

Fig. 2. Vaccine response in the booster group and full vaccination group.

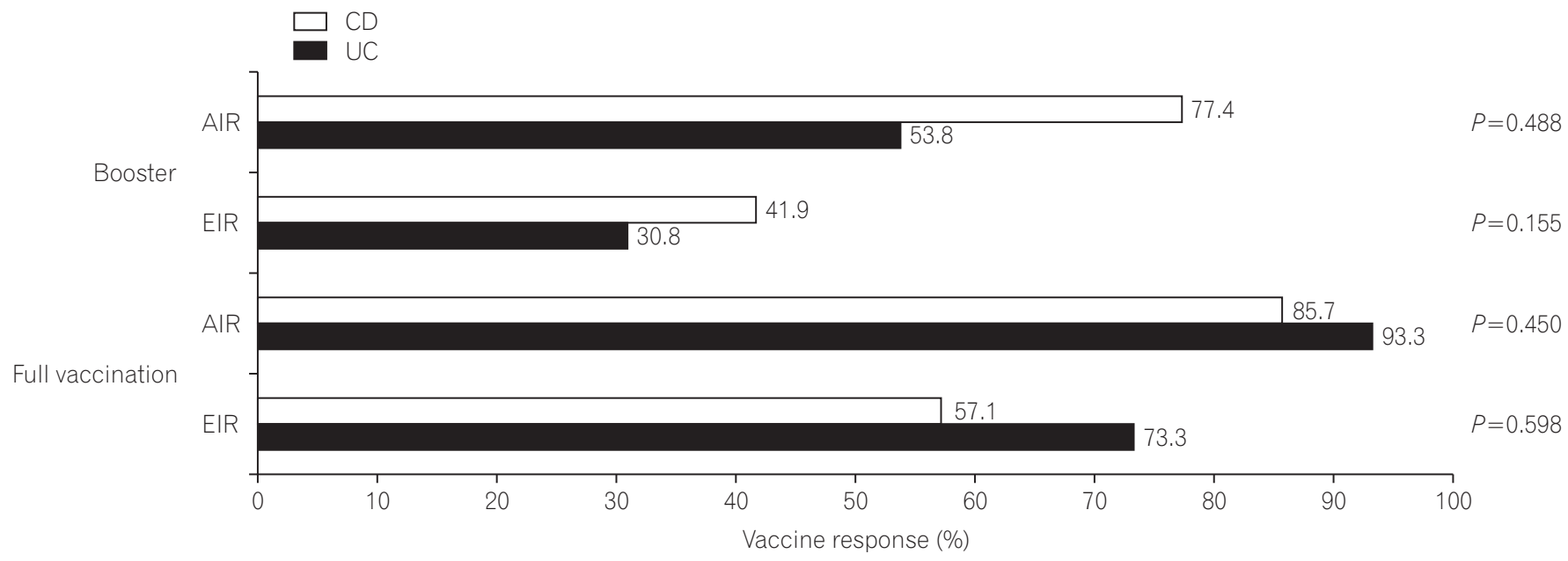

Fig. 3. Evaluation of vaccination response based on serum anti-HBs titer. AIR, adequate immune response defined as anti-HBs titer $\geq 10 \mathrm{IU} / \mathrm{L} ; \mathrm{EIR}$, effective immune response defined as anti-HBs titer $\geq 100 \mathrm{lU} / \mathrm{L}$. 
significant (Fig. 3).

To identify predictive factors for vaccine response, the associations between baseline clinical characteristics of enrolled patients and optimal vaccine response were analyzed. In univariate analysis, there were no statistically significant predictive factors. However, in a multivariate analysis that included variables that were considered as clinically significant, a younger age at the initial vaccination dose ( $<26$ years) was a positive predictor for optimal vaccine response (OR, 6.01; 95\% CI, 1.15-31.32; $P=0.033$ ). In addition, a previous complete vaccination history (OR, 0.15 ; $95 \%$ CI, $0.03-0.80$; $P=0.026$ ) was a negative predictive factor for optimal vaccine response (Table 2).

\section{Additional Full Dose Vaccine Response in Booster Vaccine Non-responders}

Among the 44 patients who received a booster vaccination, a total of 13 (UC 6, CD 7) failed to achieve optimal vaccine response. These patients were recommended to receive 2 more dose vaccinations. The mean age of this population was 28.3 years, and 8 patients (61.5\%) were male. Eleven patients $(84.6 \%)$ had remission to mild disease severity and were taking the following categories of medications: 2, biologics monotherapy or combination therapy; 7 , immunomodula-

Table 2. Clinical Factors Predictive of Optimal Vaccination Response

\begin{tabular}{|c|c|c|c|c|c|c|c|}
\hline \multirow{2}{*}{ Variable } & \multirow{2}{*}{ Total } & \multicolumn{3}{|c|}{ Univariate } & \multicolumn{3}{|c|}{ Multivariate } \\
\hline & & Non-response & Optimal $^{a}$ & $P$-value & OR & $95 \% \mathrm{Cl}$ & $P$-value \\
\hline Age at HBV vaccination $(y r)^{b}$ & & & & 0.798 & & & \\
\hline$<26$ & $34(46.6)$ & 7 (20.6) & $27(79.4)$ & & 6.01 & $1.15-31.32$ & 0.033 \\
\hline$\geq 26$ & $39(53.4)$ & $9(23.1)$ & $30(76.9)$ & & (Reference) & & \\
\hline Sex & & & & 0.656 & & & \\
\hline Male & $49(67.1)$ & $10(20.4)$ & 39 (79.6) & & & & \\
\hline Female & $24(32.9)$ & $6(25.0)$ & $18(75.0)$ & & & & \\
\hline IBD type & & & & 0.616 & & & \\
\hline UC & $28(38.4)$ & $7(25.0)$ & $21(75.0)$ & & & & \\
\hline$C D$ & 45 (61.6) & $9(20.0)$ & $36(80.0)$ & & & & \\
\hline Disease activity & & & & 0.720 & & & \\
\hline Moderate to severe & 13 (17.8) & $2(15.4)$ & $11(84.6)$ & & 2.09 & $0.32-13.53$ & 0.441 \\
\hline Remission to mild & 60 (82.2) & $14(23.3)$ & $46(76.7)$ & & (Reference) & & \\
\hline Previous HBV vaccination & & & & 0.052 & & & \\
\hline Complete & 44 (60.3) & $13(29.5)$ & $31(70.5)$ & & 0.15 & $0.03-0.80$ & 0.026 \\
\hline Incomplete or unknown & 29 (39.7) & $3(10.3)$ & 26 (89.7) & & (Reference) & & \\
\hline Disease duration (mo) & & & & 0.133 & & & \\
\hline$<24$ & $25(34.2)$ & $8(32.0)$ & $17(68.0)$ & & 0.28 & $0.06-1.19$ & 0.084 \\
\hline$\geq 24$ & $48(65.8)$ & 8 (16.7) & $40(83.3)$ & & (Reference) & & \\
\hline Type of treatment & & & & 0.765 & & & \\
\hline Biologics + immunomodulator & $10(13.7)$ & $2(20.0)$ & $8(80.0)$ & & 0.20 & $0.02-2.39$ & 0.203 \\
\hline Biologics ${ }^{c}$ & $13(17.8)$ & $2(15.4)$ & $11(84.6)$ & & 0.66 & $0.07-5.81$ & 0.706 \\
\hline Immunomodulator $^{d}$ & $28(38.4)$ & $8(28.6)$ & $20(71.4)$ & & 0.24 & $0.04-1.47$ & 0.124 \\
\hline Non-immunomodator ${ }^{e}$ & $22(30.1)$ & $4(18.2)$ & 18 (81.8) & & (Reference) & & \\
\hline
\end{tabular}

Values are presented as number (\%).

${ }^{a}$ Optimal response was defined as anti-HBs titer $\geq 10 \mathrm{IU} / \mathrm{L}$.

${ }^{b}$ Age was divided at 26 years, the median age of patients.

'Biologics included infliximab, adalimumab, golimumab or any combination of these drugs with aminosalicylates.

${ }^{d}$ Immunomodulator included corticosteroids, thiopurine, or any combination of these drugs with aminosalicylates.

${ }^{e}$ Non-immunomodulator included aminosalicylate. 


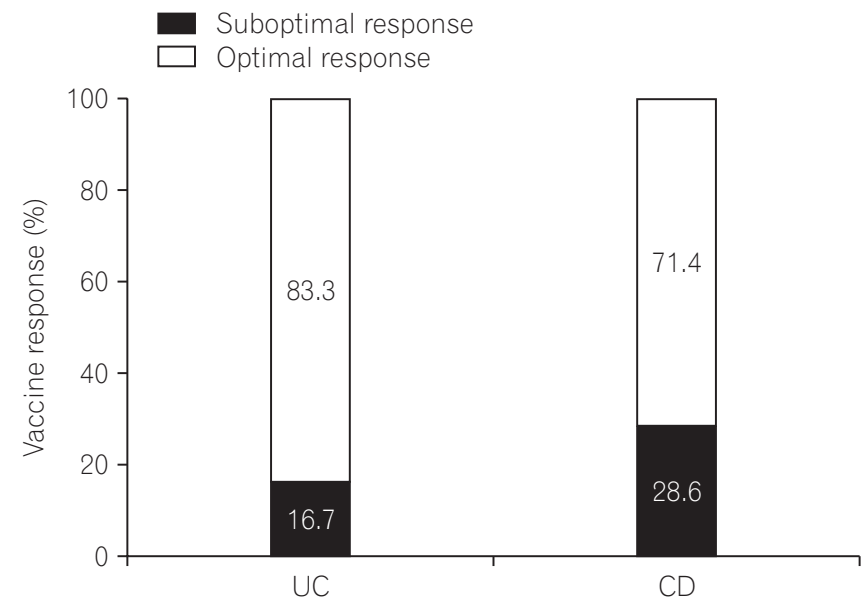

Fig. 4. Vaccine response of complete 3-dose vaccination in the patients with inadequate response after booster vaccination.

tors; and 4, non-immunomodulators. Optimal vaccine response was observed in 10 patients $(76.9 \%$, UC $83.3 \%$; CD $71.4 \%$ ) (Fig. 4).

\section{Predictive Factors Associated with Vaccination Compliance}

To identify predictive factors for compliance with the recommended vaccine schedule, the clinical characteristics of the 73 patients with good compliance and the 14 patients with poor compliance were compared. A complete previous vaccination history was the only significant factor associated with lower compliance in both univariate analysis $(P=0.029)$ and multivariate analysis (OR, $0.11 ; 95 \% \mathrm{CI}, 0.02-0.73 ; P=$ 0.022) (Table 3).

\section{DISCUSSION}

This study is the first to prospectively analyze HBV vaccine response in patients with IBD in Korea, an intermediate endemic area for HBV. We found that $26 \%$ of IBD patients did not have immunity to HBV, $49 \%$ of patients had maintained immunogenicity against HBV from a prior vaccination, and $25 \%$ of patients had prior or present HBV infection. In regard to the vaccination response, $70.5 \%$ of patients obtained an optimal response from the booster vaccination, and $89.7 \%$ of patients acquired an optimal response from the full vaccination series.

$\mathrm{HBV}$ vaccination is an effective method for reducing infection and related serious complications, including cirrhosis or hepatocellular carcinoma. In Korea, HBV vaccination prevalence has exceeded 99\% since 1990, ${ }^{17}$ which has resulted in dramatic decreases of HBsAg seropositive rates $(2.9 \%$ in the general population) ${ }^{11}$ and HBcAb seropositive rates (10.6\% in adults younger than 30 years). ${ }^{17}$ The non-immunity rate for $\mathrm{HBV}$ in the general population is reported as $19.7 \%{ }^{18}$

Compared to the general population in Korea, our study showed that IBD patients have higher risk of HBV infection and suboptimal response to HBV vaccination. Among the patients who did not have immunity, only $55 \%$ had a history of the complete vaccination series, whereas the other patients had not completed the vaccination series or the vaccination history was unknown. Recent Korean studies ${ }^{19}$ reported an HBV vaccination rate of $52.6 \%$ in IBD patients, which is higher than other vaccination rate, such as measlesmumps-rubella (42.2\%), influenza (37.5\%), varicella (34.9\%), and hepatitis A (15.6\%). However, this rate still falls short. The primary reason given for not receiving a vaccination was "not knowing the existence or necessity of vaccine." The low rate of $\mathrm{HBV}$ vaccination in IBD patients is worrisome because an endogenous immunosuppressive state arising from altered immunity and an exogenous immunocompromised state due to immunosuppressive drugs can increase the risk of opportunistic infections. ${ }^{19}$

Generally among healthy individuals, a 3-dose vaccination induces a $90 \%$ to $95 \%$ protective antibody concentration. ${ }^{20}$ Although antibody concentration wanes over time after the primary immunization, the protection can persist for at least two decades. ${ }^{21}$ Moreover, protection against disease could persist even after the disappearance of anti-HBs antibodies in individuals with an intact immune system. ${ }^{22}$ Therefore, further vaccination is usually not recommended in individuals with full response to the complete vaccination series. ${ }^{21}$

However, IBD patients have shown diminished vaccine response in the range of $33 \%$ to $76 \%^{23}$ due to aberrant Thl/ Th2 immune response, which led to disturbance of cytokine secretion and ineffective vaccine response, as well as concurrent use of immunosuppressive therapy. ${ }^{18}$ In addition, low initial antibody level was also correlated with decreased antibody persistence. ${ }^{24}$ Thus, 2017 ACG clinical guidelines recommend testing for HBV serologic markers and to vaccinate non-immune patients, particular before anti-TNF treatment. ${ }^{7}$ For patients with suboptimal antibody concentration, a single dose booster vaccine should be considered, and if the response is not optimal, a full vaccination series is recommended. ${ }^{7}$ Booster vaccinations are known to increase antibody levels rapidly by triggering memory B cells, which have been sensitized by the primary vaccine to proliferate and differentiate. ${ }^{22}$

Based on this guideline, we evaluated the response of 
Table 3. Clinical Factors Associated with Poor Compliance of HBV Vaccination Recommendation

\begin{tabular}{|c|c|c|c|c|c|c|c|}
\hline \multirow[b]{2}{*}{ Variable } & \multirow[b]{2}{*}{ Total } & \multicolumn{3}{|c|}{ Univariate } & \multicolumn{3}{|c|}{ Multivariate } \\
\hline & & $\begin{array}{c}\text { Good } \\
\text { compliance }^{a}\end{array}$ & $\begin{array}{c}\text { Poor } \\
\text { compliance }\end{array}$ & $P$-value & OR & $95 \% \mathrm{Cl}$ & $P$-value \\
\hline Age at HBV vaccination $(\mathrm{yr})^{b}$ & & & & 0.454 & & & \\
\hline$<26$ & 39 (44.8) & 34 (87.2) & $5(12.8)$ & & 2.74 & $0.41-18.28$ & 0.299 \\
\hline$\geq 26$ & $48(55.2)$ & 39 (81.3) & 9 (18.8) & & (Reference) & & \\
\hline Sex & & & & $>0.999$ & & & \\
\hline Male & $59(67.8)$ & 49 (83.1) & $10(16.9)$ & & & & \\
\hline Female & $28(32.2)$ & $24(85.7)$ & $4(14.3)$ & & & & \\
\hline IBD type & & & & 0.752 & & & \\
\hline UC & $34(39.1)$ & $28(82.4)$ & $6(17.6)$ & & & & \\
\hline$C D$ & $53(60.9)$ & $45(84.9)$ & $8(15.1)$ & & & & \\
\hline Disease activity & & & & $>0.999$ & & & \\
\hline Moderate to severe & $15(17.2)$ & 13 (86.7) & $2(13.3)$ & & 0.91 & $0.15-5.47$ & 0.918 \\
\hline Remission to mild & $72(82.8)$ & $60(83.3)$ & $12(16.7)$ & & (Reference) & & \\
\hline Previous HBV vaccination & & & & 0.029 & & & \\
\hline Complete & $48(55.2)$ & $44(91.7)$ & $4(8.3)$ & & 0.11 & $0.02-0.73$ & 0.022 \\
\hline Incomplete or unknown & $39(44.8)$ & $29(74.4)$ & $10(25.6)$ & & (Reference) & & \\
\hline Disease duration (mo) & & & & 0.607 & & & \\
\hline$<24$ & $32(36.8)$ & 26 (81.3) & $6(18.8)$ & & 1.71 & $0.41-7.07$ & 0.459 \\
\hline$\geq 24$ & $55(63.2)$ & $47(85.5)$ & $8(14.5)$ & & (Reference) & & \\
\hline Type of treatment & & & & 0.668 & & & \\
\hline Biologics + immunomodulator & & & & & $4 \times 10^{8}$ & 0 & 0.999 \\
\hline Biologics $^{c}$ & $26(29.9)$ & $23(88.5)$ & $3(11.5)$ & & 0.79 & $0.13-4.68$ & 0.797 \\
\hline Immunomodulator $^{d}$ & 33 (37.9) & $28(84.8)$ & $5(15.2)$ & & 1.11 & $0.24-5.18$ & 0.894 \\
\hline Non-immunomodulator ${ }^{\mathrm{e}}$ & $28(32.2)$ & $22(78.6)$ & $6(21.4)$ & & (Reference) & & \\
\hline
\end{tabular}

Values are presented as number (\%).

${ }^{a}$ Good compliance for the vaccination was defined as administration of all vaccination as recommend schedule.

${ }^{b}$ Age was divided at 26 years, the median age of patients.

'Biologics included infliximab, adalimumab, golimumab or any combination of these drugs with aminosalicylates.

IImmunomodulator included corticosteroids, thiopurine, or any combination of these drugs with aminosalicylates.

eNon-immunomodulator included aminosalicylate.

HBV vaccination in 2 groups classified by prior complete vaccine experience, namely the booster group and the full vaccination group. As expected, the response rates in both groups was lower than the levels within the general population, $70.5 \%$ versus $88 \%^{24}$ for booster group and, $89.7 \%$ versus $90 \%$ to $95 \%^{20}$ for full vaccination group, respectively. Young age was a significantly positive predictive factor for optimal vaccine response, whereas prior complete vaccination was a significantly negative factor. Disease type, duration, activity, and type of treatment at the time of vaccination were not significant factors.
Age has been known to have an inverse correlation with vaccine response. ${ }^{25-27}$ Immunosenescence, an aging process of the immune system including thymic involution, changes in cytokine production/distribution, and changes in the quality or quantity of the lymphocyte population results in poor response to vaccination. ${ }^{28,29}$ Based on our results, it is necessary to evaluate the immunogenicity for HBV at the diagnosis of $\mathrm{IBD}^{30}$ and to minimize the time to vaccination.

Based on the immunologic principles of vaccinations, a positive relationship between a prior complete vaccination and vaccine response would be reasonable. However, our 
result showed a negative relationship. This negative relationship should be further analyzed based on the time of the primary vaccination and the serum titers of antibodies after primary vaccination, because there could be patients who failed to respond to the primary vaccination. Nearly $5 \%$ of individuals have been reported to show blunted immune response to vaccination. ${ }^{31}$ However, nearly none of the patients in our study could recall that information.

Another explanation for this negative relationship could arise from the study design. We considered patients who were uncertain of a previous vaccination as a vaccine naïve group. Thus, some of these patients could have had a previous complete vaccination series. However, if we compare HBV vaccination rates from another Korean study, which reported a $52.6 \%$ rate, this kind of recall bias would be negligible. Fortunately, $77.2 \%$ of patients with a suboptimal response to the booster vaccine responded to an additional full series vaccine. Thus, a full series vaccination should be administered if the booster vaccination fails. Also, physicians should pay strict attention to this population of patients since a complete vaccination history is associated with poor compliance.

Immunosuppressive therapy, including conventional immunosuppressants such as corticosteroids or azathioprine, and biologics are closely related to suboptimal vaccine response. ${ }^{32,33}$ The most recent American study also reported that exposure to anti-TNF, especially infliximab was associated with decreased antibody response ${ }^{34}$ in IBD patients. However, in our study, there was no statistically significant association between the type of treatment and vaccine response although $70 \%$ of patients were under immunosuppressive therapy. These inconsistent results could be explained by different study design between 2 studies. Although the American study included relatively large number of patients, this study included patients with and without documented vaccination history which has significant influence on vaccine response as well as medication. In addition, the lack of an association between the treatment modality and response could be due to the mild disease activity of a majority of patients in our study. Therefore, we still have to monitor the negative influence of immunosuppressive agents carefully, especially the effects of anti-TNF agents on vaccine response, and consider appropriate vaccinations for non-immune patients before starting anti-TNF therapies. ${ }^{7,35,36}$

This study has several limitations. First, as a single-center study, our subjects did not necessarily represent the characteristics of the general Korean IBD patient population. Second, we considered patients "not knowing about the vaccina- tion" as non-vaccinated individuals. Some patients who had completed the vaccination, but could not recall having been vaccinated might be assigned to the full vaccination group. However, since the Korean National Immunization Program had started in 1995 and due to the relatively young age of enrolled patients, recall bias might not have significantly influenced the results. Despite these limitations, this study is valuable in terms of evaluating the status of immunogenicity against $\mathrm{HBV}$ in IBD patients within an intermediate endemic area and the vaccine response in subgroups classified as booster and vaccine naïve groups.

In conclusion, this study confirmed that IBD patients who are more susceptible to HBV infection have suboptimal response to an $\mathrm{HBV}$ vaccination. Thus, it is necessary to evaluate the immunogenicity to $\mathrm{HBV}$ at the diagnosis of IBD, and a vaccine schedule should be created as soon as possible. Considering that a previous complete HBV vaccination series is associated with suboptimal response and poor compliance, serum-HBV titers should be thoroughly evaluated in these patients, and, if negative, an additional 3-dose vaccination should be administered.

\section{FINANCIAL SUPPORT}

The authors received no financial support for the research, authorship, and/or publication of this article.

\section{CONFLICT OF INTEREST}

No potential conflict of interest relevant to this article was reported.

\section{AUTHOR CONTRIBUTION}

Conceptualization: SAJ. Methodology: SAJ, JYC. Formal analysis: JYC, CMM, SEK, KNS, HKJ. Project administration: SAJ. Writing-original draft: JYC. Writing - review and editing: JYC, SAJ. Approval of final manuscript: all authors.

\section{REFERENCESRE}

1. Altunöz ME, Senateş E, Yeşil A, Calhan T, Ovünç AO. Patients with inflammatory bowel disease have a lower response rate to HBV vaccination compared to controls. Dig Dis Sci 2012; 57:1039-1044.

2. Huang ML, Xu XT, Shen J, Qiao YQ, Dai ZH, Ran ZH. Prevalence and factors related to hepatitis B and C infection in inflammatory bowel disease patients in China: a retrospective study. J Crohns Colitis 2014;8:282-287. 
3. Gisbert JP, Chaparro M, Esteve M. Review article: prevention and management of hepatitis $\mathrm{B}$ and $\mathrm{C}$ infection in patients with inflammatory bowel disease. Aliment Pharmacol Ther 2011;33:619-633.

4. Bernal I, Domènech E, García-Planella E, Cabré E, Gassull MA. Opportunistic infections in patients with inflammatory bowel disease undergoing immunosuppressive therapy. Gastroenterol Hepatol 2003;26:19-22.

5. Michel M, Duvoux C, Hezode C, Cherqui D. Fulminant hepatitis after infliximab in a patient with hepatitis B virus treated for an adult onset still's disease. J Rheumatol 2003;30:1624-1625.

6. Wei SC, Chang TA, Chao TH, et al. Management of ulcerative colitis in Taiwan: consensus guideline of the Taiwan Society of Inflammatory Bowel Disease. Intest Res 2017;15:266-284.

7. Farraye FA, Melmed GY, Lichtenstein GR, Kane SV. ACG clinical guideline: preventive care in inflammatory bowel disease. Am J Gastroenterol 2017;112:241-258.

8. Moses J, Alkhouri N, Shannon A, et al. Hepatitis B immunity and response to booster vaccination in children with inflammatory bowel disease treated with infliximab. Am J Gastroenterol 2012;107:133-138.

9. Kim H, Shin AR, Chung HH, et al. Recent trends in hepatitis B virus infection in the general Korean population. Korean J Intern Med 2013;28:413-419.

10. Lee DH, Kim JH, Nam JJ, Kim HR, Shin HR. Epidemiological findings of hepatitis B infection based on 1998 National Health and Nutrition Survey in Korea. J Korean Med Sci 2002;17:457462.

11. Lee H, Lee H, Cho Y, Oh K, Ki M. Changes in seroprevalence of hepatitis $B$ surface antigen and epidemiologic characteristics in the Republic of Korea, 1998-2013. Epidemiol Health 2015;37: e2015055. doi: 10.4178/epih/e2015055.

12. Cho YK, Song BC. Prevention of viral hepatitis and vaccination. Korean J Med 2012;82:123-133.

13. López-Serrano P, Pérez-Calle JL, Sánchez-Tembleque MD. Hepatitis B and inflammatory bowel disease: role of antiviral prophylaxis. World J Gastroenterol 2013;19:1342-1348.

14. Ben Musa R, Gampa A, Basu S, et al. Hepatitis B vaccination in patients with inflammatory bowel disease. World J Gastroenterol 2014;20:15358-15366.

15. Lee YJ, Cheon JH, Kim JH, et al. Clinical efficacy of beclomethasone dipropionate in Korean patients with ulcerative colitis. Yonsei Med J 2017;58:144-149.

16. Sandborn WJ, Feagan BG, Hanauer SB, et al. A review of activity indices and efficacy endpoints for clinical trials of medical therapy in adults with Crohn's disease. Gastroenterology 2002;122:512-530.
17. Park NH, Chung YH, Lee HS. Impacts of vaccination on hepatitis B viral infections in Korea over a 25-year period. Intervirology 2010;53:20-28.

18. Kim ES, Cho KB, Park KS, et al. Prevalence of hepatitis-B viral markers in patients with inflammatory bowel disease in a hepatitis-B-endemic area: inadequate protective antibody levels in young patients. J Clin Gastroenterol 2014;48:553-558.

19. Yun HS, Min YW, Chang DK, et al. Factors associated with vaccination among inflammatory bowel disease patients in Korea. Korean J Gastroenterol 2013;61:203-208.

20. Coates T, Wilson R, Patrick G, André F, Watson V. Hepatitis B vaccines: assessment of the seroprotective efficacy of two recombinant DNA vaccines. Clin Ther 2001;23:392-403.

21. Poorolajal J, Hooshmand E. Booster dose vaccination for preventing hepatitis B. Cochrane Database Syst Rev 2016;(6):CD008256. doi: 10.1002/14651858.CD008256.pub3.

22. Leuridan E, Van Damme P. Hepatitis B and the need for a booster dose. Clin Infect Dis 2011;53:68-75.

23. Gisbert JP, Chaparro M. Vaccination strategies in patients with IBD. Nat Rev Gastroenterol Hepatol 2013;10:277-285.

24. Bruce MG, Bruden D, Hurlburt D, et al. Antibody levels and protection after hepatitis B vaccine: results of a 30-year follow-up study and response to a booster dose. J Infect Dis 2016;214:1622.

25. Cossio-Gil Y, Martínez-Gómez X, Campins-Martí M, et al. Immunogenicity of hepatitis B vaccine in patients with inflammatory bowel disease and the benefits of revaccination. J Gastroenterol Hepatol 2015;30:92-98.

26. Fisman DN, Agrawal D, Leder K. The effect of age on immunologic response to recombinant hepatitis B vaccine: a meta-analysis. Clin Infect Dis 2002;35:1368-1375.

27. Vida Pérez L, Gómez Camacho F, García Sánchez V, et al. Adequate rate of response to hepatitis $\mathrm{B}$ virus vaccination in patients with inflammatory bowel disease. Med Clin (Barc) 2009; 132:331-335.

28. Fattal-German M. Immunocompetence in the elderly. Ann Pharm Fr 1992;50:13-24.

29. Kumar R, Burns EA. Age-related decline in immunity: implications for vaccine responsiveness. Expert Rev Vaccines 2008; 7:467-479.

30. Kim ES. Inflammatory bowel disease is no longer a risk factor of viral hepatitis infection in Asia. Intest Res 2017;15:5-6.

31. Walayat S, Ahmed Z, Martin D, Puli S, Cashman M, Dhillon S. Recent advances in vaccination of non-responders to standard dose hepatitis B virus vaccine. World J Hepatol 2015;7:25032509 . 
32. Agarwal N, Ollington K, Kaneshiro M, Frenck R, Melmed GY. Are immunosuppressive medications associated with decreased responses to routine immunizations? A systematic review. Vaccine 2012;30:1413-1424.

33. Gisbert JP, Villagrasa JR, Rodríguez-Nogueiras A, Chaparro M. Efficacy of hepatitis B vaccination and revaccination and factors impacting on response in patients with inflammatory bowel disease. Am J Gastroenterol 2012;107:1460-1466.

34. Pratt PK Jr, David N, Weber HC, et al. Antibody response to hepatitis B virus vaccine is impaired in patients with inflammatory bowel disease on infliximab therapy. Inflamm Bowel Dis 2018;24:380-386.
35. Rubin LG, Levin MJ, Ljungman P, et al. 2013 IDSA clinical practice guideline for vaccination of the immunocompromised host. Clin Infect Dis 2014;58:e44-e100. doi: 10.1093/cid/cit684.

36. Rahier JF, Magro F, Abreu C, et al. Second European evidencebased consensus on the prevention, diagnosis and management of opportunistic infections in inflammatory bowel disease. J Crohns Colitis 2014;8:443-468. 